\title{
Cure a Head-Ache: Cement Gun Technique
}

\author{
G Green ${ }^{1,2}$, I Afzal ${ }^{*}$ and S Radha ${ }^{1,2}$ \\ ${ }^{1}$ Croydon University Hospital, UK \\ ${ }^{2}$ South West London Elective Orthopaedic Centre, UK
}

\begin{abstract}
Assembly of dual mobility heads in revision hip replacement surgery can be fraught with anxiety. Conventional manufacturer jigs are fiddly to use, and often only one is present in the hospital at any time. Slips or drops or problems with sterility can pose significant problems intra-operatively. Often the only solution in some instances being to delay the surgery and wait for new kit to arrive from nearby hospitals or loans from the manufacturer. In our District General Hospital, Croydon University Hospital, a single surgeon performs complex revision hip replacement surgery, and therefore multiple kit sets are not always present. We present a case in which a defective jig posed a problem. As no alternative was available, a new innovate technique using a standard cement gun was developed. We present this technique as an easy, effective alternative to conventional jigs.
\end{abstract}

\section{Introduction}

Assembling dual mobility heads in revision total hip replacement can be fraught will difficulty. Current manu facturers jigs are often tedious to use and assemble. In busy times and non-specialist orthopaedic centres, they often are not present on the general hospital shelves and require ordering or loaning from the manufacturer. Especially in District General Hospitals, these can be from loan sets, which often have holes in the coverings, which can compromise sterility. Also, the awkward nature of these kits makes them a slipping or dropping hazard. If the jig slips or components of it are dropped accidentally, there remain few ways of engaging the head components without causing significant delay to surgery or potential harm to the patient, or rethinking the operative plan altogether. Surgeons may try assembling in-si$t u$. However, this may compromise the trunnion and promote accelerated failure. They may use a head-pusher and attempt manual connection, but these are equally likely to slip and may scratch the underlying polyethylene.

\section{Case Report}

In our District General Hospital (DGH), there is a single surgeon who is able to provide a complex revision arthroplasty service.

Mrs. X, a 78-year-old female presented to hospital with recurrent THR dislocations. After assessing fitness for anaesthesia and ability to comply with rehabilitative instructions, the decision was made to revise the acetabular component and use a dual-mobility head. The complex Dual Mobility assembly kit is shown in (Figure 1).

\section{Method}

Intra-operatively, it was found that there was part of the manufacturer assembly jig missing. In our DGH, there was no second kit on the shelf, and given the, current, on-going coronavirus measures, there was limited support from company representatives. Therefore, creativity was required in order to safely and efficiently assemble the finickity equipment. Attempts at manual assembly proved insecure.

To safely complete the procedure and put together the head components, the surgeon developed a new technique with existing equipment.

Multiple cement guns are present and individually packed in even non-specialist DGHs, owing to the need to treat hip fractures with cemented hemiarthroplasty. These are relatively low cost, and if dropped or dysfunctional, easier to replace.

Cement guns have a plunger to push cement, and a solid end in which the cement container would usually sit. This lends itself beautifully to use in assembling the dual mobility head, as the outer head can be securely placed in the solid end. The plunger is attached to the smaller inner head to securely insert without risk of dropping or slipping.

*Corresponding author: I Afzal, South West London Elective Orthopaedic Centre, UK

Accepted: June 17, 2020

Published online: June 19, 2020

Citation: Green G, Afzal I, Radha S (2020) Cure a Head-Ache: Cement Gun Technique. J Orthop Surg Tech 3(1):130-132 


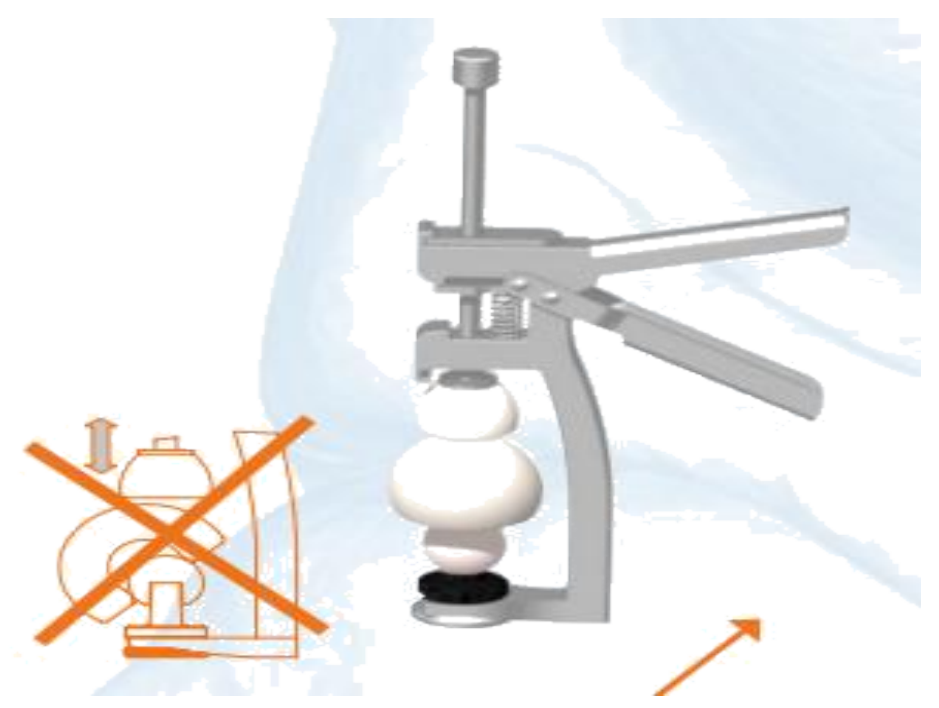

Figure 1: Example of Serf Dual Mobility head assembly kit.
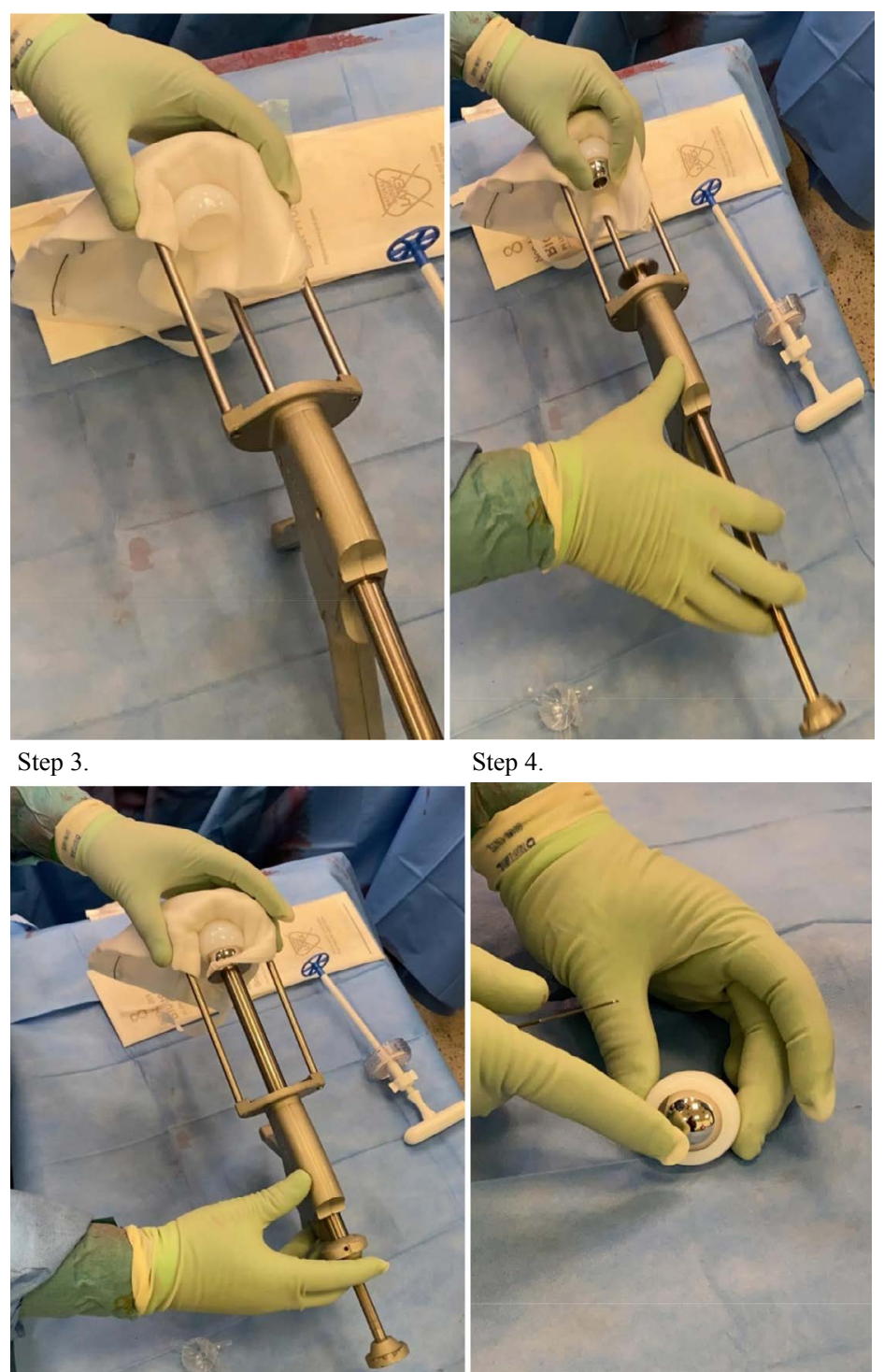

Step 4.

Step 3.

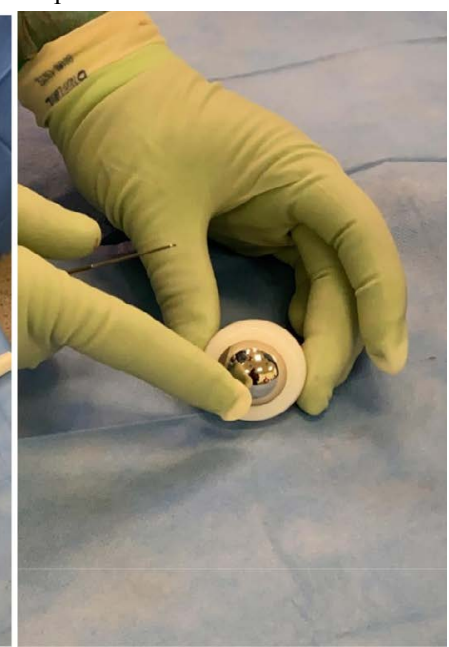

Step 4.

Figure 2: Steps in new the technique. 
The technique is shown in (Figure 2). The outer polyethylene liner is protected by a swab and placed in the end of the empty cement gun. The gun is deployed with a folded swab enveloping the metal end to protect the components and to ensure the head is square on.

The inner head is held carefully in place within the outer head and the gun is deployed, again with folded gauze protecting the components from the metal cement gun shaft until a click is heard.

The technique was developed in conjunction with theatre staff, who provided excellent feedback, quoting the ease of use and lack of need for further expensive equipment. This in- novate solution avoided an otherwise inevitable delay whilst finding alternative jigs from nearby hospitals or the company.

\section{Conclusion}

Revision of total hip replacement for dislocation with dual mobility heads is a common procedure. The manufacturer equipment for assembly can be technically difficult to use. In DGHs, there are only single kits or loan kits used which pose specific problems if dropped or are dysfunctional. We describe a new technique using readily available equipment, which is easy to use and does not require special equipment or expertise. It can be used primarily or in the case of an emergency and doesn't add additional operative time.

DOI: $10.36959 / 453 / 535$ 